\title{
Microstructure evolution during thermal processing : insight from in-situ time-resolved synchrotron radiation experiments
}

\author{
J. Bednarčík ${ }^{1,2, a}$, R. Nicula ${ }^{1, b}$, K. Saksl ${ }^{3, c}$, M. Stir ${ }^{4, d}$ and E. Burkel ${ }^{1, e}$ \\ ${ }^{1}$ Institute of Physics, Rostock University, August-Bebel-Str. 55, D-18055 Rostock, Germany \\ ${ }^{2}$ Institute of Physics, P.J. Šafárik University, Park Angelinum 9, 04154 Košice, Slovakia \\ ${ }^{3}$ Deutsches Elektronen Synchrotron (HASYLAB), Notkestr. 85, D-22607 Hamburg, Germany \\ ${ }^{4}$ ITAP, University of Stuttgart, Pfaffenwaldring 57/VI, D-70569 Stuttgart, Germany

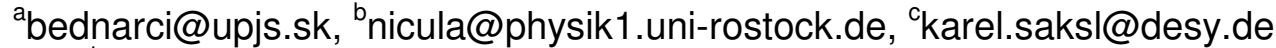 \\ dstir@itap.physik.uni-stuttgart.de, ${ }^{\mathrm{e}}$ burkel@ physik1.uni-rostock.de
}

Keywords: synchrotron radiation; in-situ X-ray diffraction; line-profile analysis; nanocrystallization; soft-magnetic nanocomposite alloys

\begin{abstract}
The magnetic, mechanical or chemical properties of nanocrystalline materials strongly differ from the ones of their coarse-grained counterparts. Moreover, significant changes of the phase diagrams were already evidenced for nanostructured alloys. Thermal processing with or without applied pressure controls the microstructure development at the nanometer scale and thus essentially decides upon the final nanomaterial behaviour and properties. A common route for the synthesis of metallic nanomaterials is the devitrification of amorphous precursors obtained via non-equilibrium processing, e.g. by rapid solidification or high-energy ball-milling. Time-resolved in-situ X-ray diffraction experiments may nowadays be performed at high-brilliance synchrotron radiation sources for a variety of temperature-pressure conditions. The temperature-time evolution of the grain-size distribution and microstrain can be monitored in detail at specimen-relevant scales. Together with local information from electron microscopy and chemical analysis, in-situ X-ray experiments offer a complete set of tools for engineering of the microstructure in nanomaterials. The effect of individual processing steps can be distinguished clearly and further tuned. An example is provided, concerning the high-temperature microstructure development in Co-rich soft magnetic nanostructured alloys.
\end{abstract}

\section{Introduction}

Modern nanostructured soft-magnetic alloys typically consist of nanocrystalline ferromagnetic grains embedded in an amorphous phase. This type of nanocomposite microstructure provides an efficient averaging of the magneto-crystalline anisotropy and magnetostrictive coefficients of the nanophase over a large number of grains within the nanomaterial.

During the past years, several Fe-based soft-magnetic nanostructured alloys were developed, e.g. $\mathrm{Fe}_{73.5} \mathrm{Si}_{13.5} \mathrm{~B}_{9} \mathrm{Nb}_{3} \mathrm{Cu}_{1}$ (FINEMET, [1]), $\mathrm{Fe}_{88} \mathrm{Zr}_{7} \mathrm{~B}_{4} \mathrm{Cu}_{1}$ (NANOPERM, [2]) and (Fe,Co) ${ }_{88} \mathrm{M}_{7} \mathrm{~B}_{4} \mathrm{Cu}_{1}$ with $\mathrm{M}: \mathrm{Zr}$, Nb or Hf (HITPERM, [3]). The recent research on soft-magnetic nanocomposites was reviewed by several authors [4-8]. The search for bulk amorphous metallic glasses with good soft magnetic properties [8], further led to the discovery of Co-rich bulk amorphous alloys in the Co-Fe$\mathrm{M}-\mathrm{B}$ alloy system ( $\mathrm{M}: \mathrm{Zr}, \mathrm{Nb}$ ) [9]. The new Co-based amorphous alloys exhibit low coercivity and stable high permeability in the frequency range up to $1 \mathrm{MHz}$. Wide supercooled liquid regions of up to $80 \mathrm{~K}$ were evidenced for Co-Fe-Zr-B alloys with Fe content in excess of 14 at.\%. Cobalt-rich alloys with nearly zero magnetostriction were as well obtained at lower Fe concentrations, near to 2 at.\% [10]. There is an increasing interest in the further development of these alloys, in view of their unique combination of soft-magnetic and mechanical properties. In-situ high-temperature X-ray diffraction using synchrotron radiation and thermal analysis were used to investigate the nano- 
crystallization of amorphous and of ball-milled $\mathrm{Co}_{56} \mathrm{Fe}_{16} \mathrm{Zr}_{8} \mathrm{~B}_{20}$ (at.\%) alloy powders. Amorphous ribbons were obtained by single-roller melt-spinning. Further powder specimens were prepared by short time ball-milling of the as-quenched ribbons. The evolution of the microstructure and of the average microstrain with temperature was obtained from the diffraction line profile analysis of $\mathrm{X}$ ray diffraction patterns collected in the high-temperature range up to $800^{\circ} \mathrm{C}$.

\section{Experimental}

Master alloys with nominal composition $\mathrm{Co}_{56} \mathrm{Fe}_{16} \mathrm{Zr}_{8} \mathrm{~B}_{20}$ (at.\%) were prepared by arc-melting of high-purity elements $(>99.8 \%)$ under protective inert Ar atmosphere. From these prealloys, ribbons (10 mm wide and $40 \mu \mathrm{m}$ thick) were prepared by single-roller melt-spinning. The as-quenched ribbons were subsequently dry milled for 12 hours in a high-energy RETSCH PM-4000 planetary ball-mill using hardened-steel vials and balls. The milling experiment was performed at a ball-topowder BPR mass ratio of 31:1 with a speed of $200 \mathrm{rpm}$. To ensure effective milling, the direction of rotation of the milling plate was changed every minute. All powder handling operations were carried out in a glove box under inert $\mathrm{Ar}$ atmosphere (level of $\mathrm{O}_{2}, \mathrm{H}_{2} \mathrm{O}<1 \mathrm{ppm}$ ). The thermal stability of the samples was analyzed by differential scanning calorimetry (DSC) using a NETZSCH DSC Pegasus $404 \mathrm{C}$ at $10^{\circ} \mathrm{C} / \mathrm{min}$ heating rate under flowing argon. The saturation magnetization $\left(M_{S}\right)$ of selected samples was determined from the hysteresis loops measured at room temperature with a maximum field $H_{\max }=1.6 \mathrm{MA} / \mathrm{m}$ with a VSM LakeShore 735 vibrating sample magnetometer. High-energy X-ray diffraction (XRD) experiments were performed at the PETRA2 HASYLAB beamline (DESY Hamburg, Germany) using monochromatic synchrotron radiation of $115 \mathrm{keV}$. The specimens were measured in transmission, with typical exposure times of $180 \mathrm{~s}$. The incident beam was collimated to a $1 \mathrm{~mm}^{2}$ cross-section. XRD patterns were recorded using a 2D mar345 image plate detector, used in asymmetric mode to obtain data at high wave vector transfer $(Q=4 \pi \sin \theta / \lambda)$. The background intensity was subtracted from the $2 \mathrm{D}$ XRD patterns and the result integrated to $Q$-space using Fit2D [11]. The integrated data were corrected for polarization, sample absorption, fluorescence contribution and inelastic scattering. The total structure factor $S(Q)$ was obtained using the Faber-Ziman equation [12]. The temperature stability of the as-quenched and high-energy ball-milled $\mathrm{Co}-\mathrm{Fe}-\mathrm{Zr}-\mathrm{B}$ specimens was as-well investigated by in-situ angulardispersive $\mathrm{X}$-ray diffraction using the high-resolution diffractometer at the $\mathrm{B} 2$ beamline in HASYLAB/DESY. The synchrotron radiation wavelength was set to $\lambda=0.47026 \AA$. Heating experiments were performed in-situ, using an image plate detector and a STOE furnace.

\section{Results and discussion}

Mechanical crystallization. Fig. 1 shows the total structure factors $(S(Q)-1)$ of the as-quenched $\mathrm{Co}_{56} \mathrm{Fe}_{16} \mathrm{Zr}_{8} \mathrm{~B}_{20}$ ribbons and of powder samples ball-milled (BM) for different times. The asquenched sample exhibits a diffuse scattering pattern typical for metallic glasses with a maximum at $Q=3.06 \AA^{-1}$ and pronounced oscillations visible up to $Q \sim 15 \AA^{-1}$. The second broad peak at $Q \sim$ $5.2 \AA^{-1}$ has a shoulder at the high- $Q$ side which again is a common feature of structure factors of conventional metallic glasses. The $S(Q)$ of powder samples milled for 2 hours shows already several small and broad Bragg peaks of the bcc-Fe phase (marked by signs) in addition to the diffuse amorphous halo. The intensity of the bcc-Fe peaks further increases with milling time. This indicates that the originally amorphous sample gradually crystallizes during milling. After 12 hours of milling, the Fe phase peaks (marked by signs) become more pronounced, but remain rather broad (peak widths FWHM in excess of $0.2 \AA^{-1}$ ). 


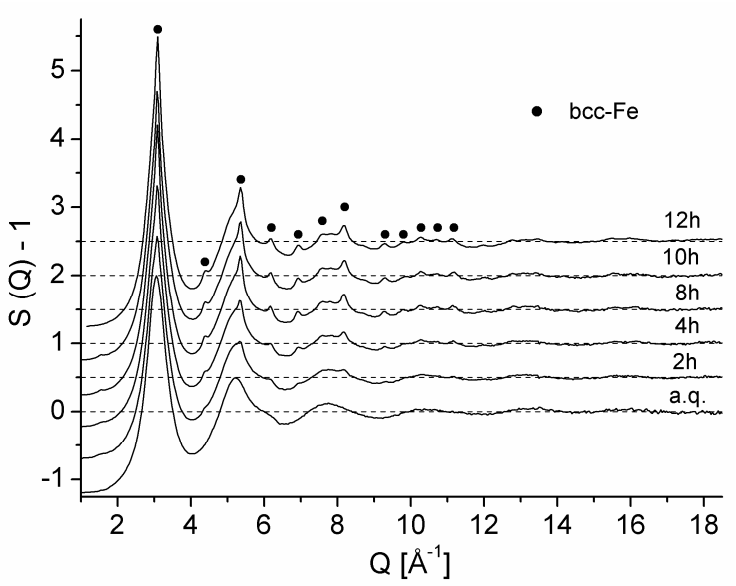

Fig. 1 Structure factors $S(Q)-1$ of the $\mathrm{Co}_{56} \mathrm{Fe}_{16} \mathrm{Zr}_{8} \mathrm{~B}_{20}$ alloy in as-quenched state and milled for different times.

This indicates that longer milling times result in an increase of crystalline phase fraction, yet the crystallites remain of nanometer size.

Thermal DSC analysis reveals the one-step crystallization of amorphous $\mathrm{Co}_{56} \mathrm{Fe}_{16} \mathrm{Zr}_{8} \mathrm{~B}_{20}$ alloys, indicated by a sharp exothermic peak with onset temperature $T_{x}=617^{\circ} \mathrm{C}$ (Fig. 2). Furthermore, the X-ray analysis (not shown) of ribbons annealed for $30 \mathrm{~min}$. at $800{ }^{\circ} \mathrm{C}$ show that crystallization occurs via the singlestage formation bcc-Fe and $\mathrm{ZrCo}_{3} \mathrm{~B}_{2}$ phases. The ball-milled powder specimens follow a similar crystallization route as the amorphous alloy ribbons. However, the exothermic heat $H_{x}$ released by the ball-milled samples upon crystallization, gradually decreases with the increasing milling time $t_{m}$ (see inset in Fig. 2).

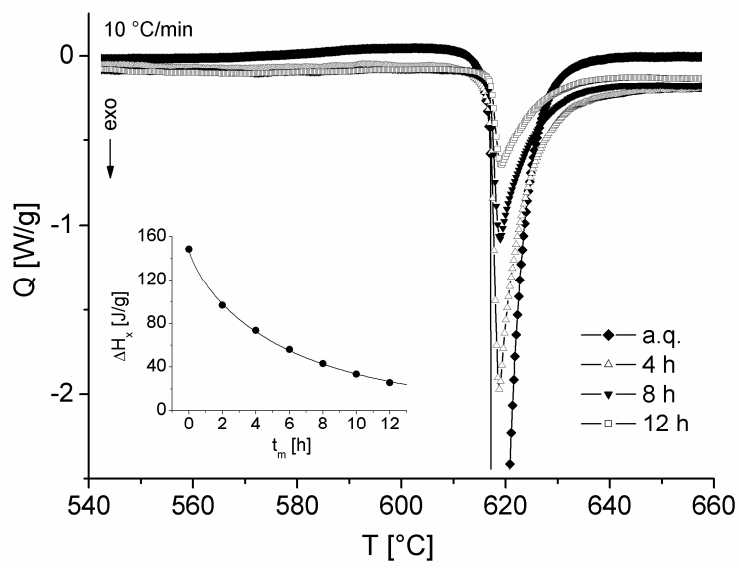

Fig. 2 DSC scans of as-quenched ribbon and powder samples milled for 4, 8, and $12 \mathrm{~h}$. The inset shows the evolution of the crystallization enthalpy $H_{x}$ with milling time $t_{m}$.

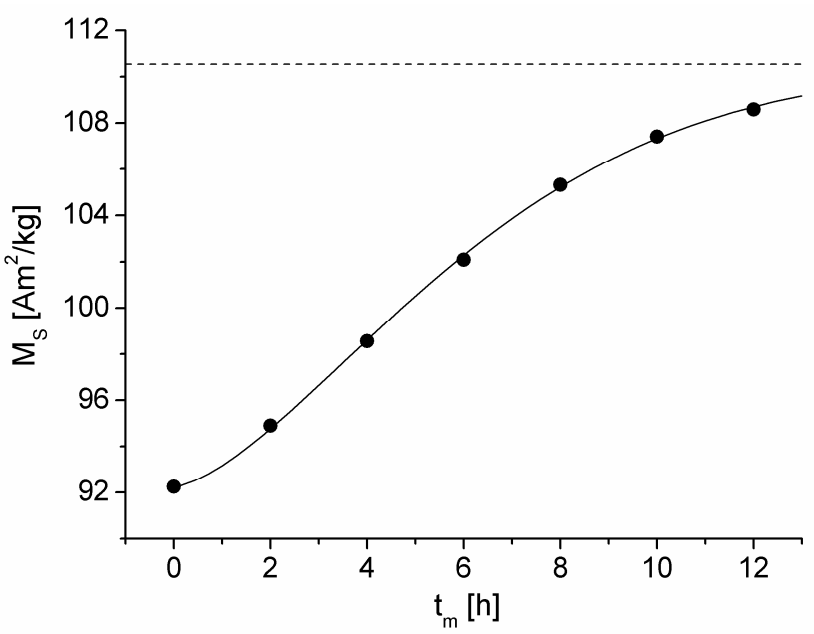

Fig. 3 The saturation magnetization $M_{S}$ as function of milling time $t_{m}$. The milling time $t_{m}=0 \mathrm{~h}$ corresponds to the asquenched ribbon. The solid line corresponds to a JohnsonMehl-Avrami fit of the data.

This result is further consistent with the increase of the crystalline bcc-Fe fraction during BM also evidenced by X-ray diffraction. Since bcc-Fe exhibits a higher $M_{S}$ than the amorphous phase, it was possible to follow the mechanically induced crystallization of the amorphous ${ }_{0}{ }_{56} \mathrm{Fe}_{16} \mathrm{Zr}_{8} \mathrm{~B}_{20}$ alloy by measuring the saturation magnetization of powder samples ball-milled for different times (Fig. 3). Assuming that the total magnetic moment after a certain milling time $t$ is a superposition of the moments of the amorphous fraction $(1-x)$ and the crystallized fraction $x$, the saturation magnetization data can be fitted by a Johnson-Mehl-Avrami model for the transformed fraction $x$

$$
\begin{aligned}
& x(t)=1-\exp \left(-k t^{\mathrm{n}}\right) \\
& M(t)=(1-x) M_{a q}+x M_{c r}
\end{aligned}
$$

where $M_{\mathrm{aq}}$ is the saturation magnetization of the amorphous ribbon and $M_{\mathrm{cr}}$ is the saturation magnetization of the mechanically crystallized sample. The parameter $k$ is a rate constant and $n$ the Avrami exponent which depends on the mechanism (nucleation and growth) of the crystallization. Since the phase composition and thus also the saturation magnetization of the thermally crystallized alloy is different from that of the alloy crystallized mechanically by $\mathrm{BM}, M_{\mathrm{cr}}$ is a free parameter of 
the fitting procedure. The least-squares fit to the data gives $k=0.051 \pm 0.003 \mathrm{~h}^{-1}, n=1.53 \pm 0.05$, and $M_{\mathrm{cr}}=110.5 \pm 0.5 \mathrm{Am}^{2} \mathrm{~kg}^{-1}$. The dashed line in Fig. 3 corresponds to $M_{\mathrm{cr}}$. The value of the exponent $n$ close to 1.5 is consistent with a zero-nucleation rate [13] and isotropic crystallite growth from very small dimensions. Friedrich et al. [14] and Trudeau et al. [15] reported for the mechanical crystallization of $\mathrm{Fe}_{86} \mathrm{Zr}_{7} \mathrm{~B}_{6} \mathrm{Cu}_{1}$ and $\mathrm{Fe}_{66} \mathrm{Co}_{18} \mathrm{Si}_{1} \mathrm{~B}_{15}$ values of $n=1.50$ and $n=1.46$, respectively. On the other hand, Guo and Lu [16] observed an Avrami exponent of $n=1.0$ for the mechanical crystallization of $\mathrm{Fe}_{77.2} \mathrm{Mo}_{0.8} \mathrm{Si}_{9} \mathrm{~B}_{13}$.

Thermal crystallization. X-ray diffraction patterns (Fig. 4a) were collected upon heating of the asquenched ribbons at constant heating rate $\left(0.3{ }^{\circ} \mathrm{C} / \mathrm{min}\right)$. The onset temperature for the crystallization of the amorphous $\mathrm{Co}_{56} \mathrm{Fe}_{16} \mathrm{Zr}_{8} \mathrm{~B}_{20}$ alloy equals $\mathrm{T}_{\mathrm{x}}=600^{\circ} \mathrm{C}$, in agreement with DSC results. The asquenched ribbons crystallize into a mixture of bcc $\mathrm{Co}(\mathrm{Fe})$ (PDF 44-1433) and hexagonal $\mathrm{ZrCo}_{3} \mathrm{~B}_{2}$ (PDF 39-0438) phases. Another in-situ heating experiment (see Fig. 4b) was performed on powders obtained by dry ball-milling of the as-quenched ribbons for 12 hours.
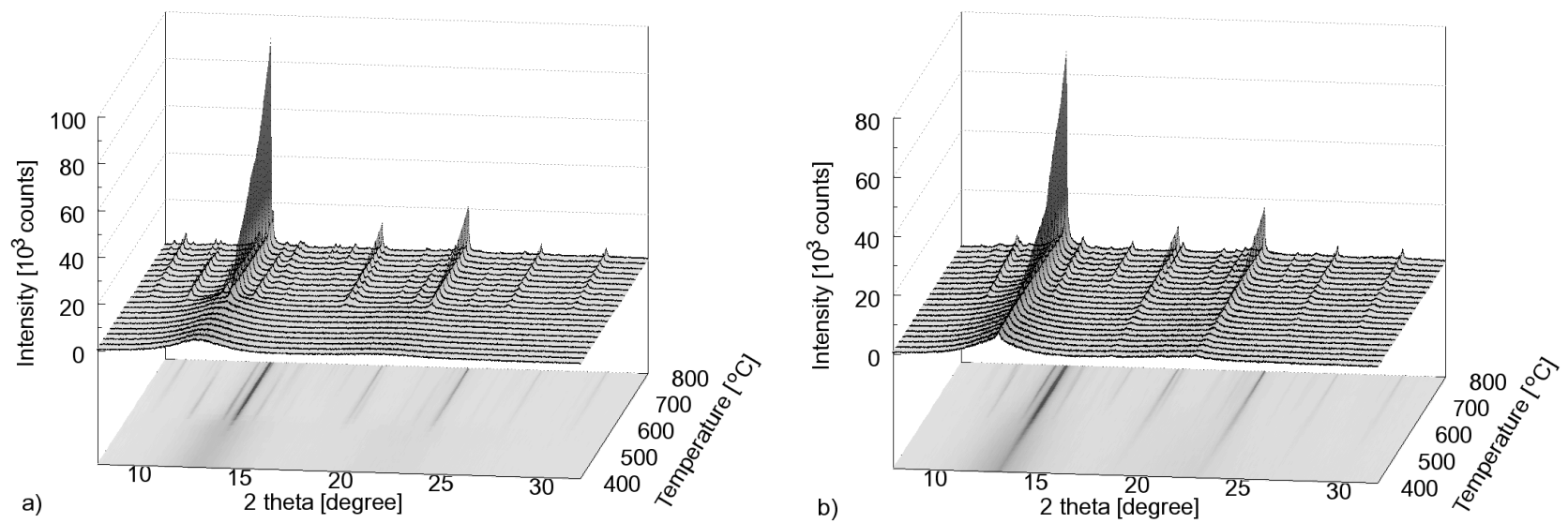

Fig. 4 Synchrotron radiation powder diffraction patterns collected during constant rate heating of $(a)$ amorphous asquenched ribbons and $(b)$ powder sample milled for $12 \mathrm{~h}$.

After the full-pattern decomposition of the XRD patterns using the PEAKFIT ${ }^{\odot}$ software package, the (110) and (220) diffraction lines of the bcc $\mathrm{Co}(\mathrm{Fe})$ nanocrystalline phase were used to infer the temperature-resolved volume-weighted grain-size, average microstrain (Fig. 5), and the grain-size distribution functions (Fig. 6). We used the double-Voigt line-profile analysis (LPA) algorithm [17].

The resulting grain-size distributions were leastsquares fitted to both log-normal and gamma distribution functions, respectively. Irrespective of temperature or of specimen type (either asquenched or ball-milled), the experimental grain-size distributions are much better described by gamma distribution functions. The resulting area normalized gamma distributions are illustrated in Fig. 6. While the evolution with temperature and the average volumeweighted grain-sizes are rather similar for both as-quenched and as-milled specimens (Fig. 5), the widths of the grain-size distributions vary significantly (Fig. 6).

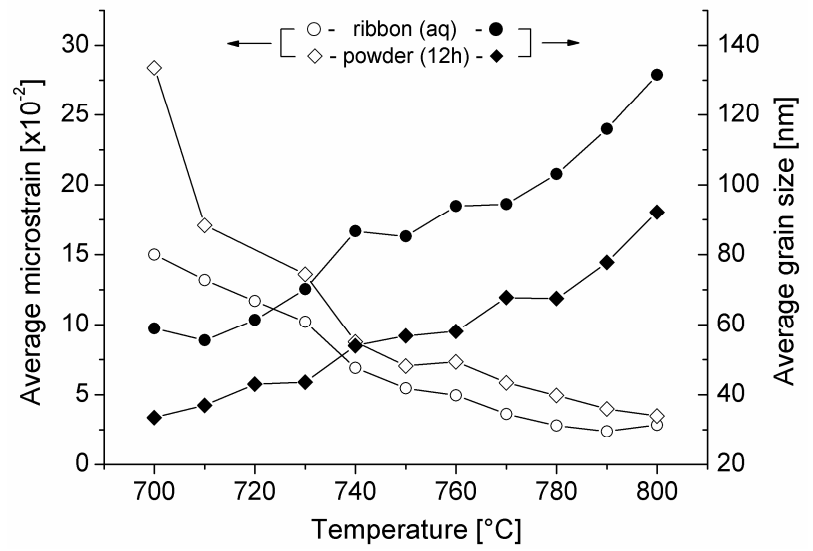

Fig. 5 Temperature evolution of the average grain size and microstrain for the bcc $\mathrm{Co}(\mathrm{Fe})$ main phase of the $\mathrm{Co}$ Fe-Zr-B alloy. 
The grain-size distribution functions are significantly narrower for the ball-milled Co-Fe-Zr-B powder specimens, which correlates with the partial crystallization during ball-milling. Cumulative grain size distributions were also retrieved (Fig. 7), yielding the fraction of grains having sizes below a certain arbitrary chosen value. For example, for the annealed ribbons one may readily conclude that while at $700^{\circ} \mathrm{C}$ about $88 \%$ of the grains are less than $100 \mathrm{~nm}$ in size, this fraction drops down to only $45 \%$ at $800^{\circ} \mathrm{C}$. One should as well notice the steeper increase of the cumulative distribution functions with temperature for the short-term ball-milled samples. This in turn indicates that, in order to retain a nanoscale microstructure, much lower annealing and/or sintering temperatures should be chosen for the BM powders than for the alloy ribbons.
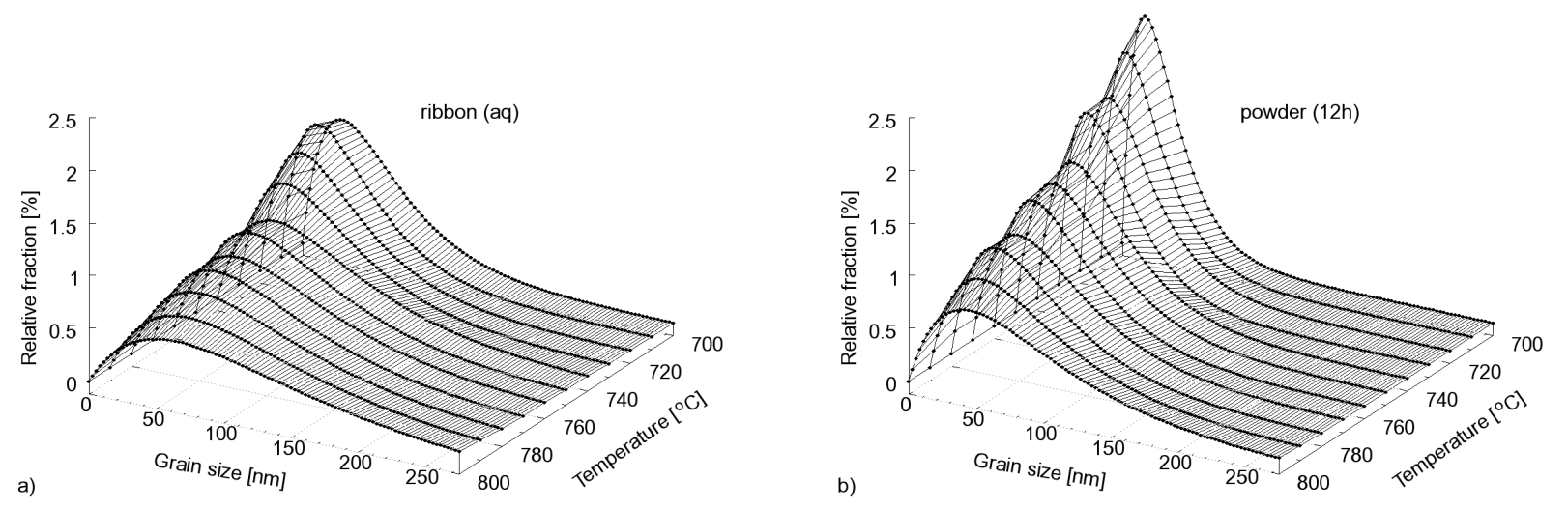

Fig. 6 Area normalized grain-size distributions vs. temperature for $(a)$ as-quenched and $(b)$ ball-milled Co-Fe-Zr-B.
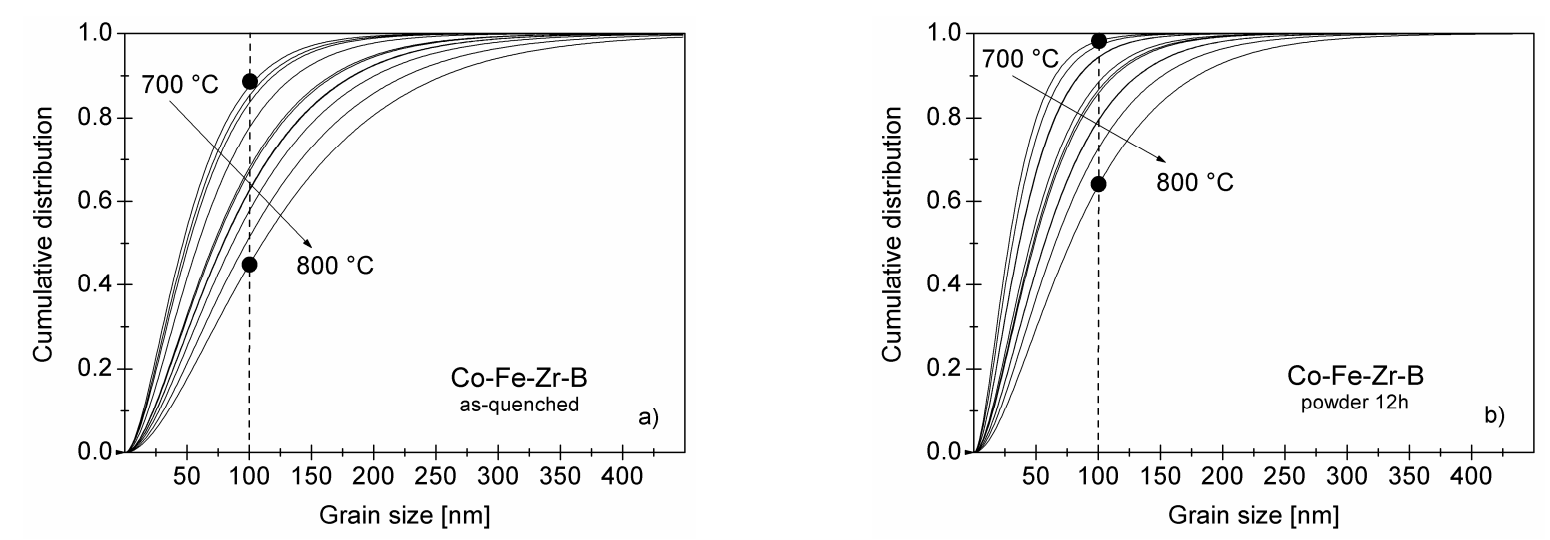

Fig. 7 Cumulative distribution functions describing the changes of the microstructure for $(a)$ alloy ribbons and $(b)$ ball milled Co-Fe-Zr-B powders upon heating to $800^{\circ} \mathrm{C}$.

The temperature- or time-dependence (coarsening kinetics) of the alloy microstructure may thus be followed in detail via the evolution of predefined crystallite-size populations (fractions) of the constituent grains. In-situ synchrotron radiation X-ray diffraction experiments therefore provide at specimen-relevant scales an accurate (yet statistical) description of the temperature-time-dependent evolution of the microstructure. Information on nanocrystallization and grain-growth is essential to the guidance of sintering experiments; for instance, sintering rates were already shown to vary significantly with the grain-size distribution widths [18].

\section{Summary}

The nanocrystallization of amorphous Co-Fe-Zr-B alloys obtained by melt-spinning was followed in detail using in-situ synchrotron radiation diffraction experiments. Both thermal- and mechanically- 
induced crystallization processes were investigated. We could evidence the one-step crystallization into a nanoscale mixture of bcc- $\mathrm{Co}(\mathrm{Fe})$ and $\mathrm{ZrCo}_{3} \mathrm{~B}_{2}$ phases at onset crystallization temperatures close to $620^{\circ} \mathrm{C}$ and the decrease of the exothermal signal with longer milling times. The use of Xray diffraction line profile analysis allowed us to monitor not only the temperature/time evolution of the average crystallite sizes and microstrains, but also the global microstructural changes of the alloy specimens as reflected by the temperature dependent grain size distribution functions. Our insitu X-ray diffraction experiments followed by line broadening examination yielded the important conclusion that short-term milling of amorphous $\mathrm{Co}_{56} \mathrm{Fe}_{16} \mathrm{Zr}_{8} \mathrm{~B}_{20}$ ribbons determines the formation of nanometer size clusters within the initial amorphous phase; the partial nanocrystallization further promotes narrower final grain-size distributions (at all temperatures) however also a more efficient coarsening of the microstructure (evidenced by the steeper rise of cumulative distribution functions with temperature). For magnetic nanocomposite materials, it is important to favour a certain specific population of crystallites of optimal size. The above results thus have important consequences on the practical choice of the thermal processing (isothermal annealing, sintering) parameters: while more homogeneous nanostructures can be obtained for the short-term ball-milled powders (with the expected improvement of the soft magnetic behaviour), the thermal treatment temperatures should be chosen much lower than for alloy ribbons, in order that a specific predefined microstructure is retained at the nanoscale.

Acknowledgements. This work was financially supported by the Marie Curie Programme of the European Community (HPMD-CT-2001-00089) and by the Slovak APVV (APVT-20-008404).

\section{References}

[1] Y. Yoshizawa, S. Oguma, K. Yamauchi, J. Appl. Phys. 64 (1998), p. 6044.

[2] K. Suzuki, A. Makino, N. Kataika, A. Inoue, T. Masumoto, Mater. Trans. JIM 32 (1991), p. 93.

[3] M.A. Willard, D.E. Laughlin, M.E. McHenry, D. Thoma, K. Sickafus, J.O. Cross, V.G. Harris, J. Appl. Phys. 84 (1998), p. 6773.

[4] M.E. McHenry, M.A. Willard, D.E. Laughlin, Prog. Mater. Sci. 44 (1999) 291.

[5] M.E. McHenry, D.E. Laughlin, Acta Mater. 48 (2000), p. 223.

[6] M.A. Willard, D.E. Laughlin, M. McHenry, J. Appl. Phys. 87 (2000), p. 7091.

[7] S. Linderoth, in : Science of Metastable and Nanocrystalline Alloys, A.R. Dinesen, M. Eldrup, D.J. Jensen, S. Linderoth, T.B. Pedersen, N.H. Pryds, A. Schroder, J.A. Pedersen (ed.), 2001, pp. 69-88.

[8] A. Inoue, Acta Materialia 48 (2000), p. 279.

[9] A. Inoue, T. Zhang, H. Koshiba, A. Makino, J. Appl. Phys. 83 (1998), p. 6326.

[10] A. Inoue, H. Koshiba, T. Itoi, A. Makino, Appl. Phys. Lett. 73 (1998), p. 744.

[11] A.P. Hammersley, S.O. Svensson, M. Hanfland, A.N. Fitch, D. Häusermann, High Press. Res., Vol. 14 (1996), p. 235.

[12] T.E. Faber and J.M. Zimman, Philos. Mag. Vol. 11 (1965), p. 153.

[13] J.W. Christian, The Theory of Transformation in Metals and Alloys (Pergamon, Oxford, United Kingdom, 1974).

[14] J. Friedrich, U. Herr, and K. Samwer, J. Appl. Phys. 87 (2000), p. 2464.

[15] M.L. Trudeau, J.Y. Huot, R. Schulz, D. Dussault, A.V. Neste, and G.L'Espérance, Phys. Rev. B 45 (1992), p. 4626.

[16] F.Q. Guo, K. Lu, Metall. Mater. Trans. A 28A (1997), p. 1123.

[17] D. Balzar, H. Ledbetter, J. Appl. Cryst. 26 (1993), p. 97.

[18] J.S. Chappell, T.A. Ring, J.D. Birchall, J. Appl. Phys. 60 (1986), p. 383. 